Rev. High Pressure Sci. Technol., Vol. 7 (1998) 481 483

\title{
Millikelvin Diamond Anvil Cell for the Study of Quantum Critical Phenomena
}

\author{
R.K.W. Haselwimmer, A.W. Tyler* and E. Pugh \\ Low Temperature Physics Group, Cavendish Laboratory, University of Cambridge, Cambridge, UK \\ * Interdisciplinary Research Centre in Superconductivity, University of Cambridge, Cambridge, UK
}

\begin{abstract}
We have developed a diamond anvil cell in which the force generation mechanism, a helium pressurised bellows, can be operated at low temperature to give in-situ pressure variation at pressures of up to $500 \mathrm{kbar}$ at millikelvin temperatures. The novel coupling of the force generation bellows to the cell thermally isolates it from the mixing chamber of the dilution fridge, as well as allowing the cell to be used in the small bore of a high field magnet. AC-susceptibility measurements have been made for the first time using a low temperature impedance matching in conjunction with high-stability electronic compensation, yielding a measure close to the true sample susceptibility.

[ low temperature, AC-susceptibility, Quantum Criticality, high-field, diamond anvil cell]
\end{abstract}

\section{Introduction}

In metals containing a lattice of $\mathrm{d}$ - or $\mathrm{f}$-electron sites the resultant strong correlations can lead to the formation of a strongly renormalised Fermi-Liquid ground state. In such a state the mass of the 'quasiparticle' charge carriers which can be up to 1000 times that of a bare electron. The strong spin correlations in these so-called HeavyFermion metals can lead to the formation of a magnetically ordered state at low temperatures, often with moments of only a fraction of $\mu_{\mathrm{B}}$. For such systems, that are naturally close to a magnetic instability, high pressure can be used to tune the magnetic ordering temperature continuously to absolute zero, where Quantum Critical behaviour [1] is expected. In such a regime, the magnetic fluctuations in the liquid of conduction quasiparticles are not quenched, as in the ordered state, but persist as critical fluctuations, producing a divergent quasiparticle-quasiparticle interaction that will ultimately yield some kind of condensation or breakdown in the Fermi-Liquid. This breakdown may be of a continuous type or very rapid, with a transition to a state with a different type of quantum order.

Conventional hydrostatic clamp cell techniques have previously been used to produce a Marginal Fermi-Liquid in the itinerant magnet $\mathrm{MnSi}$ [2], and Non-Fermi-Liquid states coupled with novel superconductivity in the Heavy-Fermions, $\mathrm{CePd}_{2} \mathrm{Si}_{2}[3]$, and more recently $\mathrm{CeNi}_{2} \mathrm{Ge}_{2}$ [4]. For studies of a wider range of materials at higher pressures we have developed a variable pressure Diamond Anvil Cell (DAC), capable of producing 500 kbar, in which the force is generated by a lowtemperature bellows pressurised by liquid helium [4]. The complete unit is mounted on a dilution fridge to reach temperatures as low as 50 millikelvin, with in-situ pressure variation allowing entire phase diagrams to be mapped in a single low-temperature experiment.

We have based our system around the commercially available Dunstan/Diacell miniature DAC [5] made of non-magnetic Beryllium-Copper (Cobalt) alloy. This cell uses a novel diamond tilt mechanism and can be operated

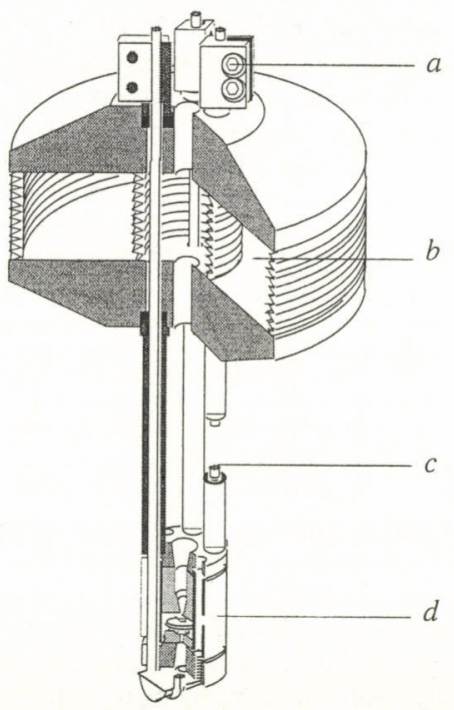

Fig. 1. Cutaway drawing of the Millikelvin Diamond Anvil Cell and cryogenic helium bellows. The force is transmitted from the bellows (b) to the DAC (d) by tension wires (c) that run through the bellows and are secured with friction clips (a).

remotely via a tensioning cables. A force of up to 1 Tonne is provided by a stainless steel bellows pressurised with liquid helium at up to $25 \mathrm{Bar}$. The double bellows design shown in figure 1 simplifies the connection to the force transfer wires, as well as providing line-of-sight access direct to the DAC. The bellows end plates are relatively free to tilt, allowing forces on opposite wires to equalise, though the friction clips are adjusted before each low-temperature run to provide initial parallelism. The $2.5 \mathrm{~mm}$ diameter stainless steel tension wires used have a breaking strain of 0.6 Tonne per wire and the friction clips have been developed to the point that each clip can reliably take over 0.35 Tonne before slippage. 
The force transfer mechanism provides significant thermal isolation between the bellows, which is held at a constant temperature of $1.5 \mathrm{~K}$, and the DAC, which can consequently be cooled to $50 \mathrm{mK}$ when thermally sunk to the mixing chamber of a dedicated dilution fridge. Extra thermal isolation has been provided by using Vespel SP22 washers mounted on the ends of the compression tubes and on spacer elements that sit beneath the friction clips.

The high pressures made available with, and the small force needed to operate, the DAC are offset by the very small samples (a maximum of $150 \mu \mathrm{m}$ square and $30 \mu \mathrm{m}$ thick) that can be pressurised. For magnetic measurements this sample volume and the fact that any susceptometer coils can only reliably be placed outside of the pressurised volume, results in a highly unfavourable signal-to-background ratio as well as the more usual difficulties associated with low signal-to-noise. Noiselevels permitting, the voltage produced by a superconducting transition in the sample is only a tiny fraction (less than one part in 300,000 ) of the voltage induced in the either of the pickup coils.

The use of balanced oppositely wound pickup coils, as is usual practise, can only improve the situation to a certain extent. Typical pairs of coils of a thousand turns can in principle only be balanced to one part in 1000 , or 1 turn, and then only by repeatedly testing the susceptometer whilst it is being wound. Although we do this routinely, it is typically only possible to achieve a balance of $0.3 \%$, which means that the signal of interest will still only be 1 part in every 1000 of background signal. The balance also changes once a gasket is used and the susceptometer is inserted into the cell, as a result of screening effects at finite frequency.

This low signal-to-background ratio is further compounded by the fact that we intend to make measurements below $30 \mathrm{~K}$, where the Curie-like paramagnetic response of magnetic impurities in materials near the susceptometer start to become appreciable. As a result the gaskets used have been made from newly available samples of ultra-pure BerylliumCopper(Cobalt) alloy [6]. This alloy contains the same ratios of elements as standard Beryllium-Copper (Cobalt) alloy 25 , but is made from ultra-pure starting materials. Importantly this alloy has almost as low a Curie tail in its magnetic susceptibility as ultra-pure BerylliumCopper binary, but has the same strength as the normal Cobalt containing ternary, some $80 \%$ higher than the binary.

The susceptometer consists of balanced, concentric pickup coils mounted close to one of the diamonds with 1001 turns of $10 \mu \mathrm{m}$ high-purity self-bonding copper wire on the inner and 674 turns on the outer coil. The excitation field, which is typically of the order of 1 Gauss at a frequency of $200 \mathrm{~Hz}$, is provided by a drive coil, mounted beneath the pickup coils, consisting of 342 turns of $40 \mu \mathrm{m}$ copper wire. No solder is used anywhere in the susceptometer to prevent contamination of the susceptive background by spurious superconducting transitions and the diamonds are bonded to their sapphire backing plates with a layer of electroplated copper [7].
The signal arising in balanced AC-susceptometer due to the transition of a perfect superconductor of volume $\Omega$, when operating at a frequency $f$, has been given by Klotz et al [8] as:

$$
V_{\text {sample }}=\pi \cdot f \cdot B \cdot \Omega\left(\frac{1}{(1-D)}\right)\left[\frac{N_{1}}{r_{1}}-\frac{N_{2}}{r_{2}}\right]
$$

Where $B$, the applied AC magnetic field, is typically 1 Gauss, and $D$ is the demagnetising factor of the sample, typically about $1 / 2$. The pickup coils can be considered to have rectangular cross-sections and effective radii of $r_{1}$ and $r_{2}$ equal to $2.5 \mathrm{~mm}$ and $3.6 \mathrm{~mm}$ respectively. For a sample volume of $10^{-13} \mathrm{~m}^{3}$ at typical drive frequencies of $200 \mathrm{~Hz}$ this gives a voltage pickup of approximately $3 \mathrm{nV}$. The signal that is usually induced in either of the pickup coils, calculated for the same set of experimental parameters, is nearer $1 \mathrm{mV}$, over 5 orders of magnitude higher.

The intrinsic noise level of this susceptometer is determined by the residual resistance of the pickup coils at low temperature, which is of the order of $30 \Omega$. This gives a Johnson noise at $4.2 \mathrm{~K}$ of $80 \mathrm{pV} / \sqrt{\mathrm{Hz}}$ and for a typical lock-in time constant of 3 seconds this will give an intrinsic Johnson noise at the pickup coils of only $12 \mathrm{pV}$ which is still some 200 times less than the expected signal from the sample.

This performance will only be achievable however if the pickup coils can be made to appear as much like the optimal noise resistance of the subsequent pre-amplifier, which is of the order of $100 \mathrm{~K} \Omega$. This noise matching can be achieved in an $\mathrm{AC}$ circuit by using an impedance matching transformer. Although such transformers are available off-the-shelf, since they can only be used at room temperatures they are not ideal for connecting to the pickup coils in the cryostat a considerable distance away, and the Johnson noise of their secondaries can also make a significant contribution to the total noise.

We have instead used a low temperature toroidal transformer, developed and built in the Cavendish [9], mounted close to the susceptometer, and held at a constant $1.5 \mathrm{~K}$. Any contribution of the Johnson noise in the secondary has been eliminated and the coupling to the pickup coils has been made much more stable. Extra primaries have been provided on this transformer to allow further compensation signals to be fed into the amplification chain at an early stage. The in-phase and quadrature compensation signals are derived from the main drive circuit using only high-stability analogue components. The intrinsic stability of this compensation system and the thermal stabilisation of the transformer and compensation circuitry have lead to the complete removal of any extrinsic background (figure 2a) leaving only a well-defined intrinsic background that can be fitted with a quadratic between 4 and $20 \mathrm{~K}$. This background is believed to arise from the phase shift that arises in the low temperature transformer as the resistance of the pickup coils change by small amounts at low temperature. After removal of this background we are left with a measure close to the true temperature dependence of the sample susceptibility (figure $2 b$ ). 


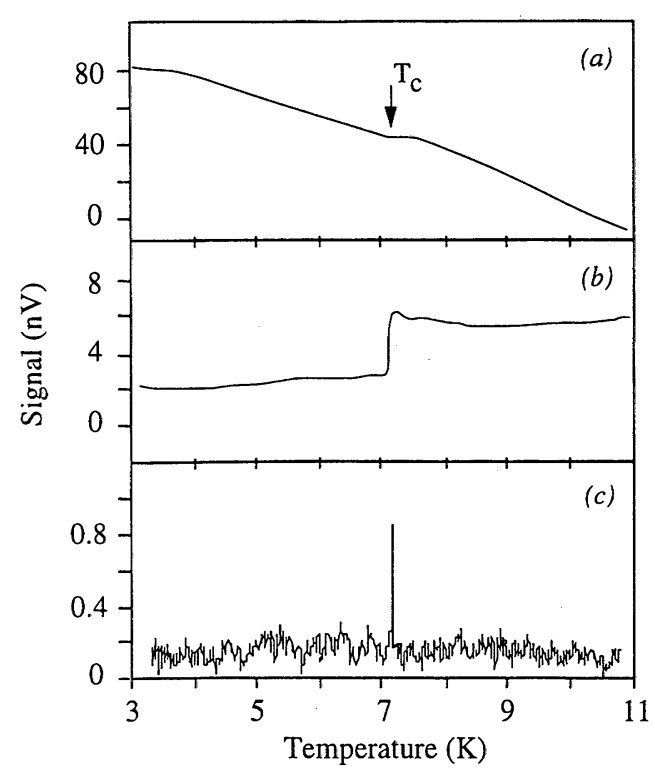

Fig. 2. Measurement of the superconducting transition of Lead by AC-susceptometry. (a) Without using any compensation, and (b) using high-stability compensation with the background subtracted. (c) The third harmonic response, though smaller in magnitude, defines the transition more precisely.

We have also developed a related technique [10] for measuring superconducting transition temperatures should they not be clearly identifiable in the fundamental signal. By detecting on the third harmonic it is possible to probe the non-linear susceptibility of the sample, and in a superconductor just below its transition temperature, the AC excitation field is enough to destroy the sample superconductivity as the field reaches a maximum each period. The magnetic susceptibility of the sample is therefore highly non-linear at this point, resulting in a peak in the third harmonic response at the transition temperature (figure $2 \mathrm{c}$.) The width of the peak in the third harmonic response, which depends on the amplitude of the excitation, can be as narrow as $10 \mathrm{mK}$ for conventional superconductors, and coincides with the traditionally determined onset of the transition to within $10 \mathrm{mK}$

\section{Acknowledgements}

This research has been undertaken in association with Professor Gilbert Lonzarich of the Cavendish Laboratory and with the generous support of various EPSRC research grants held by him. We would also like to acknowledge numerous stimulating and informative discussions with Dr. Steve Julian in Cambridge and Dr. Stan Tozer in NHFML, Tallahassee, Florida, and the technical assistance of Sam Brown in the IRC workshop in Cambridge.

\section{References}

[1] See for example the chapter by Lonzarich in 'Electron - a centenary Volume" (ed. M. Springford) Cambridge University Press, (1997)

[2] C. Pfleiderer, R.H. Friend, G.G. Lonzarich, N.R. Bernhoeft and J. Flouquet, Int. Jnl. Mod. Phys. N, 17, 887 (1993).

[3] N.D. Mathur, PhD Thesis, University of Cambridge, (1996).

[4] F.M. Grosche, S.J.S. Lister, F.V. Carter, S.S. Saxena, R.K.W Haselwimmer, N.D. Mathur, S.R. Julian and G.G. Lonzarich, Physica B 239, 62-66, (1997)

[5] D.J. Dunstan and W. Scherrer, Rev. Sci. Instrum., 59, 627-630 (1988)

[6] J.C. Cooley and M.C. Aronson, J. Alloys and Compounds (1996)

[7] M. Eremets, "High Pressure Experimental Methods", pp. 73, Oxford University Press, (1997), E. Pugh, to be published

[8] S. Klotz, J. S. Schilling and P. Muller, in "Frontiers of High-Pressure Research" (Eds. H.D. Hochheimer and R.D. Etters) Plenum Press (1991)

[9] S.R. Julian and G.G. Lonzarich, unpublished (1995)

[10] Y.A. Timofeev, High Pressure Research, 10, 504506 (1992); R.K.W. Haselwimmer, submitted to Rev. Sci. Instrum. 